\title{
LUT
}

University

\section{Automated Segmentation of the Pectoral Muscle in Axial Breast MR Images}

Zafari Sahar, Diab Mazen, Eerola Tuomas, Hanson Summer E., Reece Gregory P., Whitman Gary J., Markey Mia K., Ravi-Chandar Krishnaswamy, Bovik Alan, Kälviäinen Heikki

This is a Post-print version of a publication

published by Springer, Cham

in Advances in Visual Computing. ISVC 2019. Lecture Notes in Computer Science.

DOI: 10.1007/978-3-030-33720-9_26

Copyright of the original publication: ( ) Springer Nature Switzerland AG 2019

Please cite the publication as follows:

Zafari S. et al. (2019) Automated Segmentation of the Pectoral Muscle in Axial Breast MR Images. In: Bebis G. et al. (eds) Advances in Visual Computing. ISVC 2019. Lecture Notes in Computer Science, vol 11844. Springer, Cham

This is a parallel published version of an original publication. This version can differ from the original published article. 


\title{
Automated Segmentation of the Pectoral Muscle in Axial Breast MR Images
}

\author{
Sahar Zafari ${ }^{1,2}$, Mazen Diab ${ }^{3,4,5}$, Tuomas Eerola ${ }^{1}$, Summer E. Hanson ${ }^{5}$, Gregory P. \\ Reece $^{5}$, Gary J. Whitman ${ }^{7}$, Mia K.Markey ${ }^{4,6}$, Krishnaswamy Ravi-Chandar ${ }^{3,6}$, Alan \\ Bovik $^{2}$, and Heikki Kälviäinen ${ }^{1}$ \\ 1 Department of Computational and Process Engineering, School of Engineering Science, \\ Lappeenranta-Lahti University of Technology LUT, Finland \\ 2 Department of Electrical and Computer Engineering, The University of Texas at Austin, \\ Austin, Texas, USA \\ ${ }^{3}$ Department of Aerospace Engineering and Engineering Mechanics, The University of Texas \\ at Austin, Austin, Texas, USA \\ 4 Department of Biomedical Engineering, The University of Texas at Austin, Austin, TX, USA \\ 5 Department of Plastic Surgery, The University of Texas MD Anderson Cancer Center, \\ Houston, TX, USA \\ ${ }^{6}$ Department of Imaging Physics, The University of Texas MD Anderson Cancer Center, \\ Houston, TX, USA \\ 7 Department of Diagnostic Radiology, Division of Diagnostic Imaging, The University of \\ Texas MD Anderson Cancer Center, Houston, TX
}

\begin{abstract}
Pectoral muscle segmentation is a crucial step in various computeraided applications of breast Magnetic Resonance Imaging (MRI). Due to imaging artifact and homogeneity between the pectoral and breast regions, the pectoral muscle boundary estimation is not a trivial task. In this paper, a fully automatic segmentation method based on deep learning is proposed for accurate delineation of the pectoral muscle boundary in axial breast MR images. The proposed method involves two main steps: pectoral muscle segmentation and boundary estimation. For pectoral muscle segmentation, a model based on the U-Net architecture is used to segment the pectoral muscle from the input image. Next, the pectoral muscle boundary is estimated through candidate points detection and contour segmentation. The proposed method was evaluated quantitatively with two realworld datasets, our own private dataset, and a publicly available dataset. The first dataset includes 12 patients breast MR images and the second dataset consists of 80 patients breast MR images. The proposed method achieved a Dice score of $95 \%$ in the first dataset and $89 \%$ in the second dataset. The high segmentation performance of the proposed method when evaluated on large scale quantitative breast MR images confirms its potential applicability in future breast cancer clinical applications.
\end{abstract}

\section{Introduction}

Several studies in the literature have established the potential of patient specific biomechanical models in computing deformation of the female breast under different loading conditions, predicting outcomes of reconstructive surgeries of female breast, and 
serving as a surgical tool for image-guided lesion detection [12]3]. The basis of the biomechanical model is a patient-specific 3D geometry of the female breast that is often reconstructed from in vivo medical imaging procedures such as Magnetic Resonance Imaging (MRI). This requires segmentation of MR images to construct the 3D geometry of the female breast that is located between the front surface of the breast skin and the posterior boundary of the pectoral muscle that serves as the boundary between the breast and the rest of the body as shown in Fig. 1

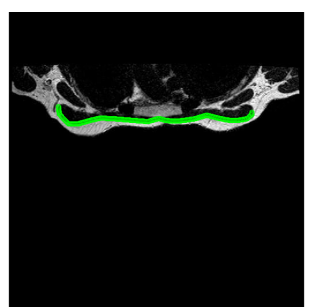

(a)

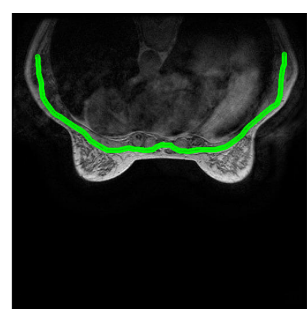

(b)

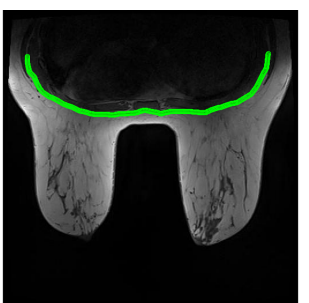

(c)

Fig. 1: Examples of breast MR images and the corresponding pectoral muscle boundaries.

Different techniques have been reported in the literature for the segmentation of the pectoral muscle and estimating its boundary [4 5[6]. Image thresholding is one of the most prominent approaches for pectoral muscle segmentation. Czaplicka et al. [7] applied an iterative Otsu's thresholding procedure with specific criteria to find the pectoral region. Mustra et al. [8] employed thresholding with image enhancement followed by polynomial curvature estimation to detect the pectoral muscle boundary. Twellmann et al. [9] used a combination of contrast enhancement, median filter, and Otsu's thresholding to extract the pectoral muscle region. The pectoral and the breast regions usually have similar image intensities, making the thresholding methods a less ideal candidate for pectoral muscle segmentation.

Gradient-based techniques are the alternative approaches for segmentation of the pectoral muscle. These methods incorporate the gradient information to estimate the boundary of the pectoral muscle. Giannini et al. [10] developed a method for detection of the upper border of the pectoral muscle using the gradient information. Chakraborty et al. [11] exploited the gradient information with curve smoothing to approximate the pectoral muscle boundary. Ferrari et al. [12] employed Gabor filters to capture pectoral muscle edges within limited orientations and computed the magnitude and phase images from each of the Gabor filter responses through a vector-summation procedure. The resulting image was used to estimate the initial pectoral boundary by Sobel edge detection. Despite all the improvements introduced by gradient-based methods, they may still experience difficulties with the segmentation of low image contrast and dense breasts. 
Line fitting is another group of methods that have been applied for the estimation of the pectoral muscle boundary. Kwok et al. [13] proposed a method that uses line fitting to highlight the pectoral muscle boundary. The estimated straight line is then refined through an iterative cliff detection [14] to estimate the actual boundary. Karssemeijer [15] combined the global thresholding and gradient information to extract the pectoral muscle region. The method utilizes Hough transform on the gradient image to represent the pectoral muscle boundary. Yam et al. [16], combined Hough transform with a dynamic programming method to approximate the pectoral muscle boundary. Nie et al. [17] proposed a method based on the B-spline curve fitting to extract and remove the pectoral muscle from the breast region. The approaches based on line fitting strongly rely on the linearity assumption of the pectoral muscle boundary, and therefore they run into difficulties when estimating the curved boundary regions of the pectoral muscle.

Atlas-based segmentation a commonly used method in medical image analysis has been also employed for the segmentation of the pectoral muscles [18]. To perform the segmentation, these methods use prior information such as shape, size, and orientation of the object. A specific model (atlas) is constructed from the prelabeled images [19]. Using an image registration technique, a coordinate mapping between an image and an atlas is computed. The end segmentation results are obtained by fusing the most similar atlases. Gubern et al. [5] developed a probabilistic atlas in a Bayesian framework for breast segmentation. Khalvati et al. [20] employed a multi-atlas segmentation algorithm that is robust to intensity variations using phase congruency maps. Fooladivanda et al. [21] introduced an atlas segmentation algorithm that uses both the pectoral muscle and the chest region. Accurate image registration and computation complexity limit the efficiency of the atlas-based methods for segmentation of the pectoral muscle.

Although the aforementioned segmentation techniques have been shown to provide satisfactory results for the segmentation of the pectoral muscle, their performance is not optimal and comes with certain limitations due to their implicit assumptions. The intensity operation-based methods (thresholding and gradient) requires accurate delineation between the pectoral muscle and the breast tissue. These methods do not work when the pectoral and the breast regions are homogeneous. Line fitting-based methods make a specific assumption about the linearity of the pectoral muscle, so, they fail to work on pectoral muscle with a curved shape. Atlas-based methods require an accurate registration algorithm and are not sufficient to provide accurate results for a wide range of datasets. In order to overcome the aforementioned challenges in pectoral muscle segmentation, a deep learning-based segmentation method is introduced in this paper that provides a more general solution for pectoral muscle segmentation in breast MR axial images.

This work makes two main contributions to the study of breast MR image segmentation. The first contribution of this work is the annotation of the pectoral muscles on MR images. To the extent of our knowledge, there is not any publicly available humanannotated dataset of the pectorals muscle on breast MR axial images. To this end, we first annotated 1100 images of 92 subjects from two different sources, 80 subjects from The Cancer Imaging Archive (TCIA) [22[23] and 12 subjects from our own private dataset, to construct a dataset for pectoral muscle segmentation. The TCIA annotation 
is publicly available at [24]. The second contribution is a segmentation method based on convolutional neural networks. The segmentation is formulated as a semantic segmentation task in which each pixel in the image is assigned to an object class, the pectoral muscle, and the background, and is implemented based on the well-known U-Net architecture [25].

After segmentation, the pectoral muscle boundary is estimated through candidate points detection and contour segmentation. Moreover, validation of existing methods is usually limited to their small private dataset of breast MRI. This work presents the first effort to expand the validation to include datasets of MR images of 92 different subjects.

\section{Proposed Method}

The proposed method automatically estimates the pectoral muscle boundary from MR axial images in two steps. First, the region of interest (ROI) i.e., pectoral muscle, is extracted from the image through a deep learning framework. Second, the pectoral muscle boundary is identified by finding the candidate points and segmenting the boundary.

Pectoral muscle segmentation: Due to heterogeneous tissues densities, neighborhood complexities and breast shape variability, segmentation of the pectoral muscle is a challenging task. In this work, pectoral muscle segmentation is formulated as a pixel classification problem that is performed by a convolutional encoding-decoding framework. In particular, it is implemented by the U-Net architecture proposed in [25]. The U-Net (see Fig. 2) contains several layers of convolutional encoders and decoders, followed by the final pixelwise classification layer. Each encoder layer is composed of duplicated $3 \times 3$ convolution operations followed by a rectified linear unit (ReLU). Following that, the encoder layers downsample the feature maps using a $2 \times 2$ max pooling operation with stride 2 . To avoid loss of spatial information during downsampling, the encoder feature maps are up-sampled and summed to the corresponding decoder feature maps and passed to the next layer after rectification in the decoder layers. To reduce the complexity and dimensionality of feature maps, the bottleneck is built from 2 convolutional layers between the encoder and decoder paths. The final layer is $1 \times 1$ convolution to map each feature vector to the desired classes. To classify each pixel and to ensure that all predicted pixels are in the range $[0,1]$ the sigmoid activation function is applied at the output layer. The loss function for training the network is based on the Dice coefficient [26]. Given the prediction $O_{p}$ and the ground truth $O_{g}$ the Dice Similarity Coefficient (DSC) measures the similarity as follows:

$$
\mathrm{DSC}=\frac{2\left|O_{p} \cap O g\right|}{\left|O_{p}\right|+|O g|} .
$$

The higher the DSC value, the greater the similarity. Since the training aims to minimize the loss function, we instead used the negative dice coefficient (-DSC).

Pectoral muscle boundary estimation: Once the pectoral muscle is segmented and its boundary is obtained by the Canny edge detection, the minimum and maximum points are identified along with the $\mathrm{X}$ coordinates of the pectoral muscle boundary. These points divide the boundary into two segments, upper and lower, as shown in 


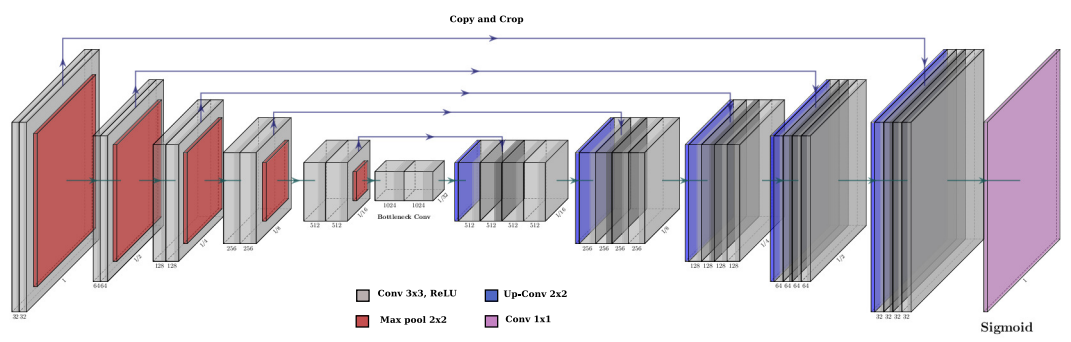

Fig. 2: U-Net architecture.

Fig. 3 b). The pectoral muscle boundary points can be found by probing the Y coordinate values of the boundary points. The pectoral muscle boundary of interest is the boundary segments with the lower average Y values.

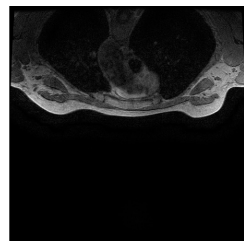

(a)

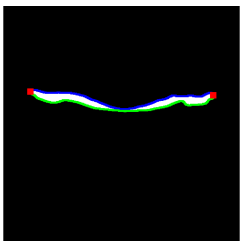

(b)

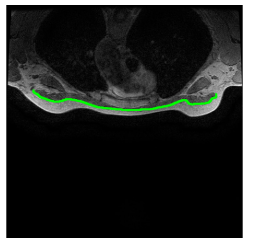

(c)

Fig. 3: Proposed method workflow:(a) Original image; (b) Segmented pectoral muscle; (c) Estimated pectoral muscle boundary.

\section{Experiments}

\subsection{Data}

The experiments were carried out on the breast MR images obtained from two sources:

The University of Texas MD Anderson Cancer Center: MR images were collected and deidentified for patients who provided informed consent in accordance with the institutional review board-approved protocol (2015-1117). This dataset contains the T1-Weighted MR images of 12 female patients, each of 512x512 pixels. The images were acquired on different GE Medical systems including Signa HDxt, Discovery MR 450 and Discovery MR750w with TR/TE $\approx 5.3 / 2.1 \mathrm{~ms}$ and bandwidth per pixel fixed at $244.1 \mathrm{HZ}$ (T1-Weighted). In total 780 images, were annotated from this dataset for training purpose.

The Cancer Imaging Archive (TCIA): Publicly available MR image datasets were obtained from the TCIA website [23]. This dataset contains the T2-Weighted MR images of 80 patients. The images were acquired using Phillips Achieva $1.5 \mathrm{~T}$ with TR/TE 
$\approx 4000 / 120 \mathrm{~ms}$ and bandwidth per pixel in the range 146-245 HZ (T2-Weighted). In total 320 images, 4 from each subject, were annotated from this dataset for testing purposes only.

The in-plane pixel resolution for the complete datasets varies between $0.48 \mathrm{~mm}$ and $0.71 \mathrm{~mm}$ and the slice thickness between $1.8 \mathrm{~mm}$ and $2.2 \mathrm{~mm}$. The weight of subjects varies from 50 to $140 \mathrm{~kg}$ with mean value of $80 \mathrm{~kg}$. To generate the binary mask and to annotate the images the Liablabel tool [27] was used. It is a polygon annotation tool that allows the user to specify semantic classes and to export these polygon files to semantic and instance label maps. This information was further used to generate the ground truth pectoral muscle boundary. A sample image and the corresponding annotation of the pectoral muscle is shown Fig. 4 .

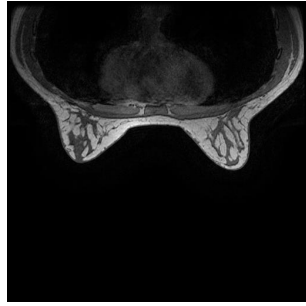

(a)

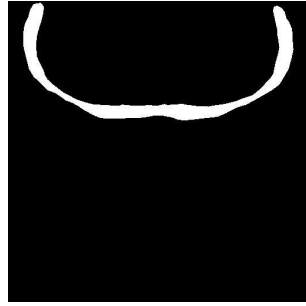

(b)

Fig. 4: An example of image and annotation mask: (a) Original image; (b) Annotation mask.

\subsection{Training}

We trained and evaluated our network on 780 images of the MD Anderson dataset. The training images were zero-centered and normalized to unit variance. The network was trained for 50 epochs using the Adam optimization algorithm with a fixed initial rate of $1 \mathrm{e}-4$ and a mini-batch size of 16 patches in Keras.

\subsection{Performance Metrics}

To evaluate the performance of the proposed segmentation method, the following metrics were used:

$$
\begin{array}{r}
\text { Recall }=\frac{\mathrm{TP}}{\mathrm{TP}+\mathrm{FN}}, \\
\text { Precision }=\frac{\mathrm{TP}}{\mathrm{TP}+\mathrm{FP}}, \\
\text { F-measure }=\frac{2 \cdot(\text { Recall } \cdot \text { Precision })}{(\text { Recall }+ \text { Precision })},
\end{array}
$$


where true positive (TP) is the number of pixels correctly segmented as pectoral muscle, false positive (FP) is the number of pixels falsely segmented as pectoral muscle, and false negative (FN) is the number of pixels falsely detected as background. To quantitatively validate the segmentation results, the Dice similarity coefficient defined in Eq. 1 was also used. DSC was chosen since it interprets the similarity by the ratio of overlap between the ground truth pectoral muscle and the segmented pectoral muscle.

To precisely evaluate the performance of automated pectoral muscle boundary detection, the average distance (AD) between the detected boundary points and their corresponding closest points on the ground truth boundary was measured and used as the boundary estimation performance metric.

\subsection{Results}

The proposed segmentation method was applied to estimate the pectoral muscle boundary in both the MD Anderson and the TCIA datasets.

To ensure the robustness and accuracy of the method, we performed 10-fold crossvalidation where the best performing model was recorded on each epoch.

The results of 10-fold cross validation for the pectoral muscle segmentation applied to the MD Anderson dataset is presented in Table 1 The results show that the proposed method achieved a Dice score of $95 \%$, precision of $94 \%$, recall of $96 \%$, and F-measure of $95 \%$.

We further evaluated the generality of the proposed method by applying it to the TCIA dataset. As it can bee seen from Table 2, the proposed method achieved a Dice score of $89 \%$, precision of $89 \%$, recall of $90 \%$, and F-measure of $89 \%$.

From Tables 1 and 2 it can be seen that the average distance between the ground truth boundary and the segmented boundary was 0.01 pixel in the MD Anderson dataset and 0.05 pixel in the TCIA dataset. This confirms the high efficiency of the proposed method in estimation of the pectoral muscle boundary.

Figs. 5 and 6 demonstrate the results of the pectoral muscle boundary detection applied to six subjects from the MD Anderson and the TCIA datasets respectively. As it can be seen, the proposed method detects the pectoral boundaries in the both datasets accurately.

Fig. 7 represents an example of a 3D point cloud and its corresponding 3D surface constructed using the segmented boundary of the pectoral muscle of 256 slices of MR images of one subject. The 3D surface is constructed using the commercial software SOLIDWORKS.

Table 1: Average performance via 10-fold cross-validation on the MD Anderson dataset.

\begin{tabular}{lccccc}
\hline Dataset & $\begin{array}{c}\text { Recall } \\
{[\%]}\end{array}$ & $\begin{array}{c}\text { Precision } \\
{[\%]}\end{array}$ & $\begin{array}{c}\text { F-measure } \\
{[\%]}\end{array}$ & $\begin{array}{c}\text { DSC } \\
{[\%]}\end{array}$ & $\begin{array}{c}\text { AD } \\
{[\text { pixel] }}\end{array}$ \\
\hline MD Anderson & $96 \pm 0.003$ & $94 \pm 0.001$ & $95 \pm 0.002$ & $95 \pm 0.002$ & $0.01 \pm 0.03$ \\
\hline
\end{tabular}



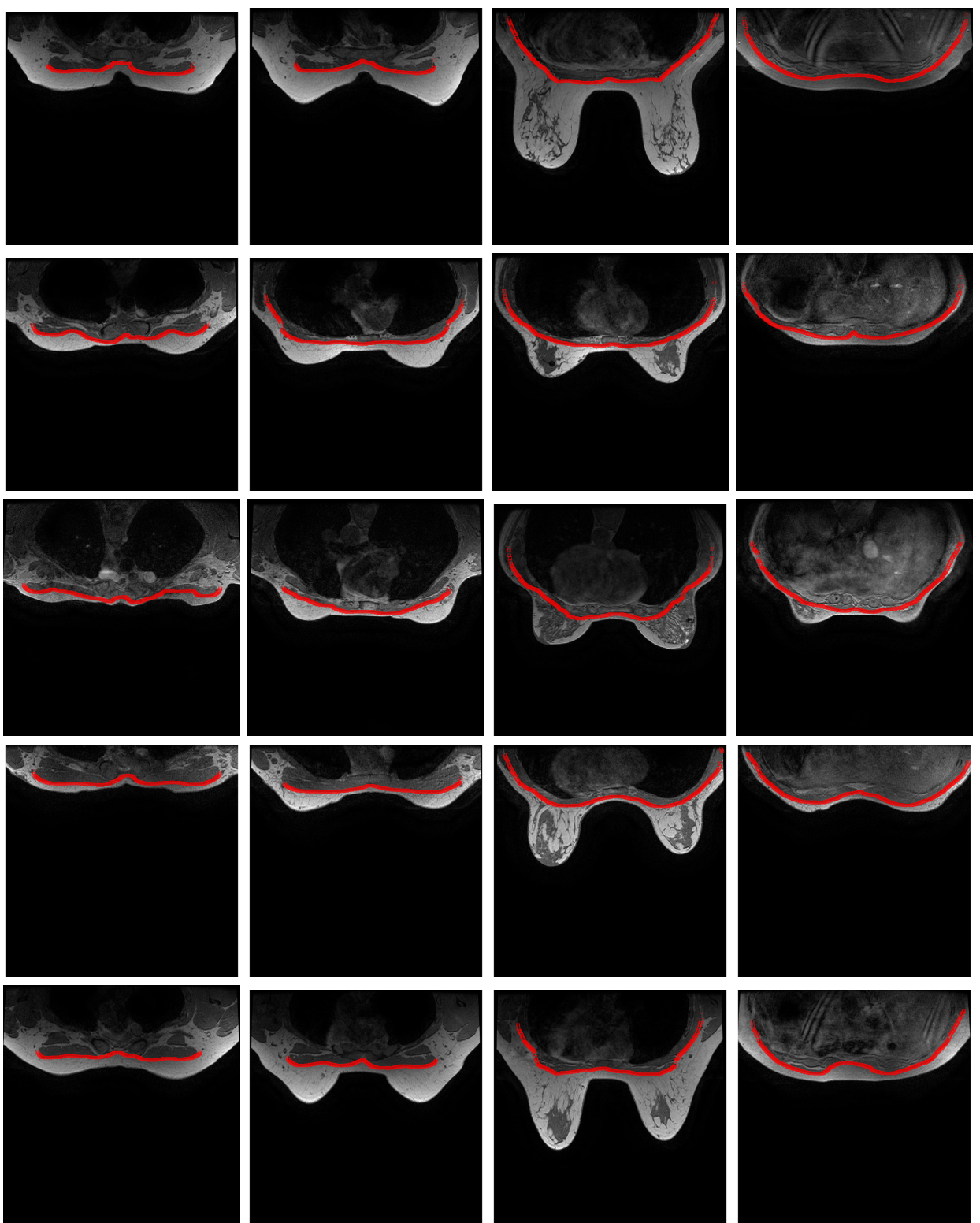

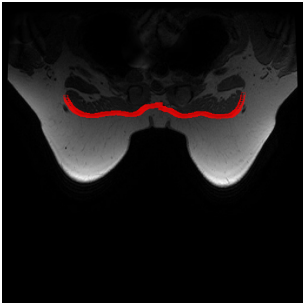

(a)

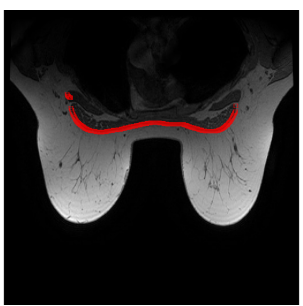

(b)

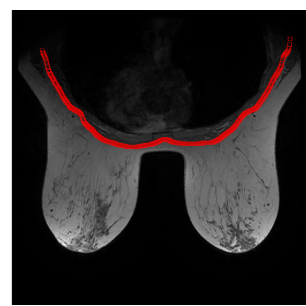

(c)

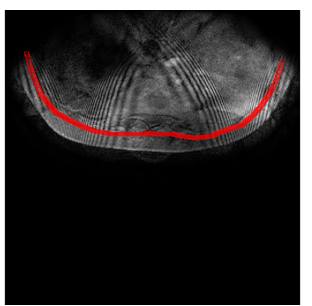

(d)

Fig. 5: Exemplar pectoral muscle boundary estimation results for six different subjects in the MD Anderson dataset having different ranges of size, shape, and weight. Each row represent the results for one subject: (a) Upper breast; (b) Superior upper breast; (c) Middle breast; (d) Lower breast. 

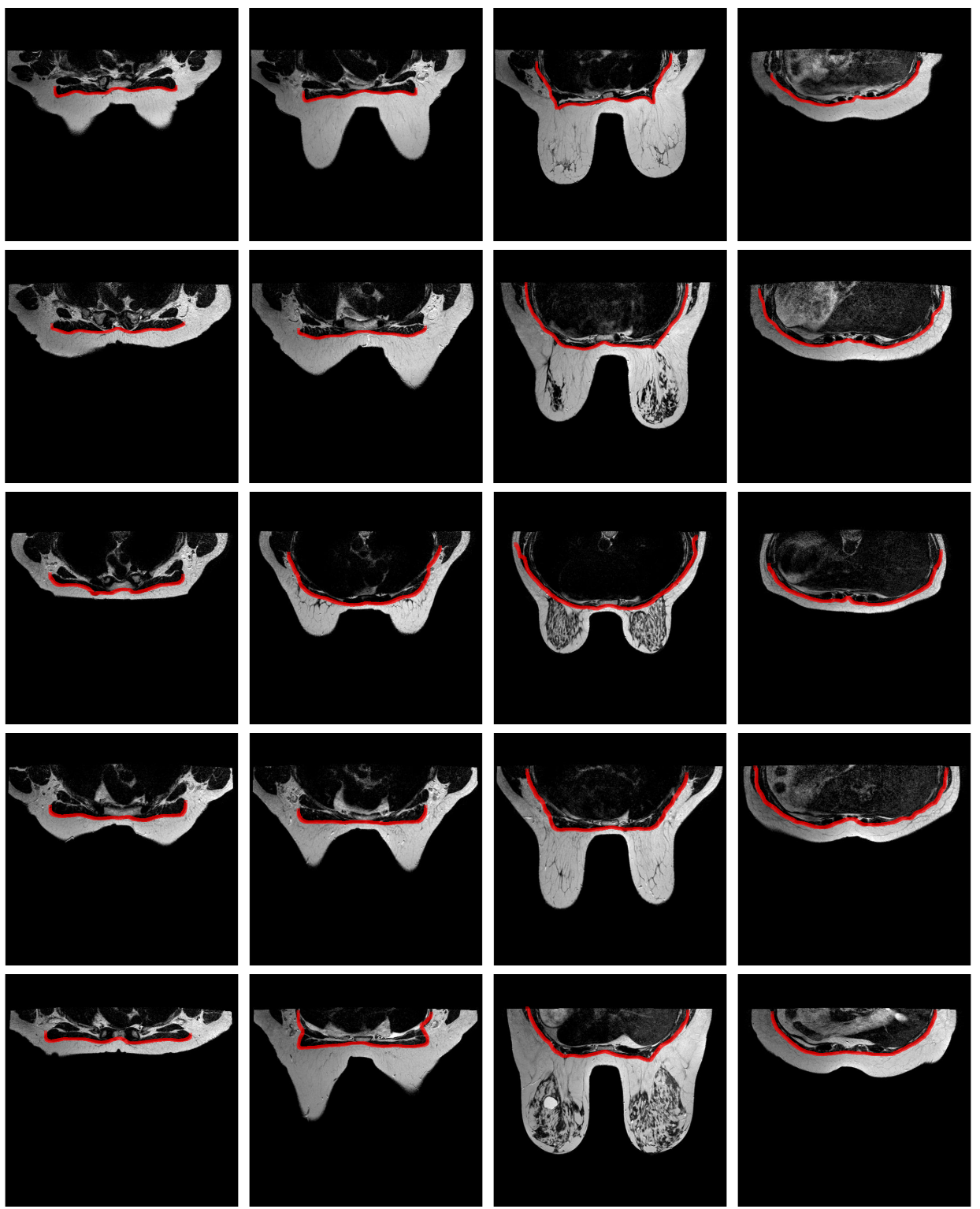

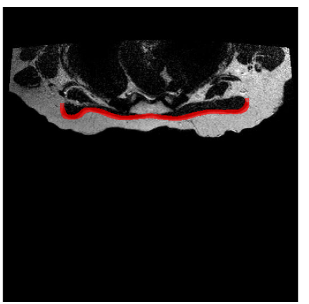

(a)

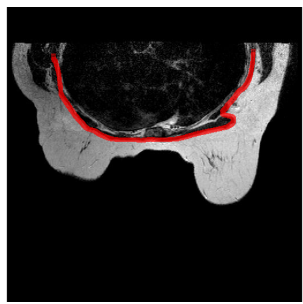

(b)

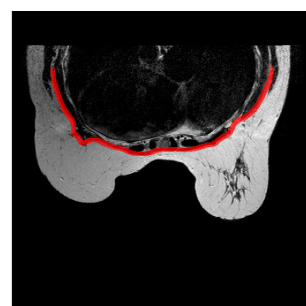

(c)

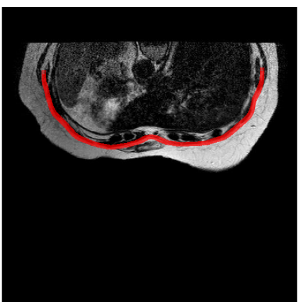

(d)

Fig. 6: Exemplar pectoral muscle boundary estimation results for six different subjects in the TCIA dataset having different ranges of size, shape, and weight. Each row represent the results for one subject: (a) Upper breast; (b) Superior upper breast; (c) Middle breast; (d) Lower breast. 
Table 2: Performance of the proposed method on the TCIA dataset when trained on the MD Anderson dataset.

\begin{tabular}{lccccc}
\hline Dataset & $\begin{array}{c}\text { Recall } \\
{[\%]}\end{array}$ & $\begin{array}{c}\text { Precision } \\
{[\%]}\end{array}$ & $\begin{array}{c}\text { F-measure } \\
{[\%]}\end{array}$ & $\begin{array}{c}\text { DSC } \\
{[\%]}\end{array}$ & $\begin{array}{c}\text { AD } \\
{[\text { pixel] }}\end{array}$ \\
\hline TCIA & $90 \pm 0.01$ & $89 \pm 0.01$ & $89 \pm 0.01$ & $89 \pm 0.04$ & $0.05 \pm 0.03$ \\
\hline
\end{tabular}

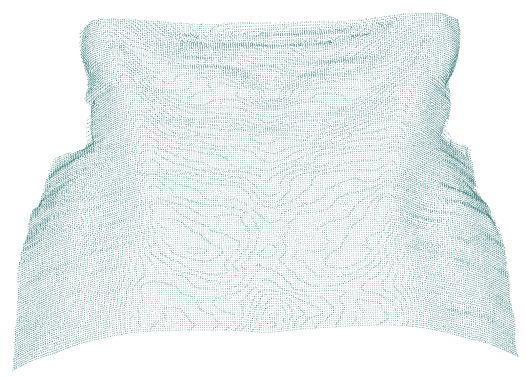

(a)

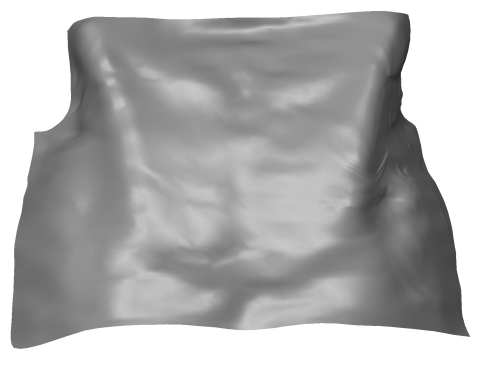

(b)

Fig. 7: A representative example of a 3D model constructed using the segmented boundary of the pectoral muscle: a) Point cloud; (b) The corresponding 3D surface.

\section{Conclusions}

This paper presents a novel method for automated segmentation and boundary delineation of the pectoral muscle in breast MR images. The proposed method consists of two steps: the pectoral muscle segmentation to segment the pectoral muscle region from the image and the pectoral muscle boundary estimation to detect the actual pectoral muscle boundary. The pectoral muscle segmentation was performed using the well known U-Net deep encoder-decoder framework. The boundary estimation was performed by detecting candidate points and contour segmentation. The proposed method was extensively evaluated on a large and challenging dataset and was shown to achieve a high segmentation accuracy.

\section{Acknowledgments}

This project was supported in part by the Academy of Finland (Cell vision project, Decision No. 313598); and The National Institutes of Health (R01CA143190 and R01CA203984). This study was approved by The University of Texas MD Anderson Cancer Center (protocol number 2015-1117). The authors would like to acknowledge the help received from Mary Catherine Bordes at The University of Texas MD Anderson Cancer Center for collecting the MRI datasets. 


\section{References}

1. Del Palomar, A.P., Calvo, B., Herrero, J., López, J., Doblaré, M.: A finite element model to accurately predict real deformations of the breast. Medical engineering \& physics 30 (2008) 1089-1097

2. Vavourakis, V., Eiben, B., Hipwell, J.H., Williams, N.R., Keshtgar, M., Hawkes, D.J.: Multiscale mechano-biological finite element modelling of oncoplastic breast surgery-numerical study towards surgical planning and cosmetic outcome prediction. PloS one 11 (2016) e0159766

3. Conley, R.H., Meszoely, I.M., Weis, J.A., Pheiffer, T.S., Arlinghaus, L.R., Yankeelov, T.E., Miga, M.I.: Realization of a biomechanical model-assisted image guidance system for breast cancer surgery using supine mri. International journal of computer assisted radiology and surgery 10 (2015) 1985-1996

4. Lei Wang, Konstantinos Filippatos, O.F.H.K.H.: Fully automated segmentation of the pectoralis muscle boundary in breast $\mathrm{mr}$ images (2011)

5. Gubern-Mérida, A., Kallenberg, M., Marti, R.M., Karssemeijer, N.: Segmentation of the pectoral muscle in breast mri using atlas-based approaches. Medical image computing and computer-assisted intervention : MICCAI ... International Conference on Medical Image Computing and Computer-Assisted Intervention 15 Pt 2 (2012) 371-8

6. Pandey, D., Yin, X., Wang, H., Su, M.Y., Chen, J.H., Wu, J., Zhang, Y.: Automatic and fast segmentation of breast region-of-interest (roi) and density in mris. Heliyon 4 (2018) e01042

7. Czaplicka, K., Włodarczyk, H., et al.: Automatic breast-line and pectoral muscle segmentation. Schedae Informaticae 2011 (2012) 195-209

8. Mustra, M., Grgic, M.: Robust automatic breast and pectoral muscle segmentation from scanned mammograms. Signal processing 93 (2013) 2817-2827

9. Twellmann, T., Lichte, O., Nattkemper, T.W.: An adaptive tissue characterization network for model-free visualization of dynamic contrast-enhanced magnetic resonance image data. IEEE Transactions on Medical Imaging 24 (2005) 1256-1266

10. Giannini, V., Vignati, A., Morra, L., Persano, D., Brizzi, D., Carbonaro, L., Bert, A., Sardanelli, F., Regge, D.: A fully automatic algorithm for segmentation of the breasts in dce-mr images. In: 2010 Annual International Conference of the IEEE Engineering in Medicine and Biology Society, EMBC'10. (2010) 3146-3149

11. Chakraborty, J., Mukhopadhyay, S., Singla, V., Khandelwal, N., Bhattacharyya, P.: Automatic detection of pectoral muscle using average gradient and shape based feature. Journal of digital imaging 25 (2012) 387-399

12. Ferrari, R.J., Rangayyan, R.M., Desautels, J.E.L., Borges, R.A., Frere, A.F.: Automatic identification of the pectoral muscle in mammograms. IEEE Transactions on Medical Imaging 23 (2004) 232-245

13. Sze Man Kwok, Chandrasekhar, R., Attikiouzel, Y., Rickard, M.T.: Automatic pectoral muscle segmentation on mediolateral oblique view mammograms. IEEE Transactions on Medical Imaging 23 (2004) 1129-1140

14. Kwok, S.M., Chandrasekhar, R., Attikiouzel, Y.: Automatic pectoral muscle segmentation on mammograms by straight line estimation and cliff detection. In: The Seventh Australian and New Zealand Intelligent Information Systems Conference, 2001. (2001) 67-72

15. Karssemeijer, N.: Automated classification of parenchymal patterns in mammograms. Physics in Medicine and Biology 43 (1998) 365-378

16. Yam, M., Brady, M., Highnam, R., Behrenbruch, C., English, R., Kita, Y.: Three-dimensional reconstruction of microcalcification clusters from two mammographic views. IEEE Transactions on Medical Imaging 20 (2001) 479-489 
17. Nie, K., Chen, J.H., Chan, S., Chau, M.K.I., Yu, H.J., el Bahri, S., Tseng, T., Nalcioglu, O., $\mathrm{Su}$, M.Y.: Development of a quantitative method for analysis of breast density based on three-dimensional breast mri. Medical physics 3512 (2008) 5253-5262

18. Gubern-Mérida, A., Wang, L., Kallenberg, M., Martí, R., Hahn, H.K., Karssemeijer, N.: Breast segmentation in mri: quantitative evaluation of three methods. In: Medical Imaging 2013: Image Processing. (2013) 86693g-86693g-7

19. Cabezas, M., Oliver, A., Lladó, X., Freixenet, J., Cuadra, M.B.: A review of atlas-based segmentation for magnetic resonance brain images. Computer methods and programs in biomedicine 104 (2011) e158-e177

20. Khalvati, F., Gallego-Ortiz, C., Balasingham, S., Martel, A.L.: Automated segmentation of breast in 3-d $\mathrm{mr}$ images using a robust atlas. IEEE Transactions on Medical Imaging 34 (2015) 116-125

21. Fooladivanda, A., Shokouhi, S.B., Mosavi, M.R., Ahmadinejad, N.: Atlas-based automatic breast mri segmentation using pectoral muscle and chest region model. In: 2014 21th Iranian Conference on Biomedical Engineering (ICBME). (2014) 258-262

22. Clark, K., Vendt, B., Smith, K., Freymann, J., Kirby, J., Koppel, P., Moore, S., Phillips, S., Maffitt, D., Pringle, M., et al.: The cancer imaging archive (tcia): maintaining and operating a public information repository. Journal of digital imaging 26 (2013) 1045-1057

23. Bloch and, B. Nicolas and, J.a.A.a., Jaffe, , C.C.: Data from breast-diagnosis. The Cancer Imaging Archive. (2015)

24. Zafari, S., Eerola, T., Kälviäinen, H.: Cellvision - automatic segmentation of overlapping objects for cell image analysis, the cell vision project web page. (http://www2.it. lut.fi/project/cellvision/index.shtml)

25. Ronneberger, Olafand Fischer, P., Brox, T.: U-net: Convolutional networks for biomedical image segmentation. In: Medical Image Computing and Computer-Assisted Intervention. (2015)

26. Zhang, W., Li, R., Deng, H., Wang, L., Lin, W., Ji, S., Shen, D.: Deep convolutional neural networks for multi-modality isointense infant brain image segmentation. NeuroImage $\mathbf{1 0 8}$ (2015) $214-224$

27. Geiger, A., Lauer, M., Wojek, C., Stiller, C., Urtasun, R.: 3d traffic scene understanding from movable platforms. Pattern Analysis and Machine Intelligence (PAMI) (2014) 